\title{
La construction de la parole du personnage dans le texte en cours de production
}

\section{The construction of characters' speech during the text} production process

\section{La construcción del discurso del personaje en el texto que se está produciendo}

\section{Lidiane Lira et Eduardo Calil}

Volume 19, numéro 3, 2016

La litéracie à l'école : regard pluridisciplinaire sur l'apprentissage de la lecture et de l'écriture de la maternelle à la fin du primaire

URI : https://id.erudit.org/iderudit/1045176ar

DOI : https://doi.org/10.7202/1045176ar

Aller au sommaire du numéro

Éditeur(s)

Faculté d'éducation, Université de Sherbrooke

ISSN

1911-8805 (numérique)

Découvrir la revue

Citer cet article

Lira, L. \& Calil, E. (2016). La construction de la parole du personnage dans le texte en cours de production. Nouveaux cahiers de la recherche en éducation, 19(3), 32-54. https://doi.org/10.7202/1045176ar
Résumé de l'article

Cet article décrit les cas de discours rapporté (DR) pendant la production de contes étiologiques inventés par une dyade d'élèves (sept ans). Nous mettrons en évidence les types de DR pour un ensemble de dix manuscrits et nous illustrerons la genèse du DR d'un processus d'écriture, depuis sa formulation initiale jusqu'à sa textualisation. Dans le cadre théorique de la génétique textuelle et de la linguistique d'énonciation, nous verrons le contexte de la rédaction pendant sa construction, en observant le rôle de chacun des élèves. Nous soulignerons que le nombre plus important de DR, 113 à l'oral pour 29 à l'écrit, semble être lié à la complexité des formes graphiques et énonciatives du DR à l'écrit, étant donné la perte de leurs possibilités d'énonciation. 


\title{
La construction de la parole du personnage dans le texte en cours de production
}

\author{
Lidiane Lira \\ Université Fédérale d'Alagoas, Brésil \\ Eduardo Calil \\ Université Fédérale d'Alagoas, Brésil
}

\section{Résumé}

Cet article décrit les cas de discours rapporté (DR) pendant la production de contes étiologiques inventés par une dyade d'élèves (sept ans). Nous mettrons en évidence les types de DR pour un ensemble de dix manuscrits et nous illustrerons la genèse du DR d'un processus d'écriture, depuis sa formulation initiale jusqu'à sa textualisation. Dans le cadre théorique de la génétique textuelle et de la linguistique d'énonciation, nous verrons le contexte de la rédaction pendant sa construction, en observant le rôle de chacun des élèves. Nous soulignerons que le nombre plus important de DR, 113 à l'oral pour 29 à l'écrit, semble être lié à la complexité des formes graphiques et énonciatives du DR à l'écrit, étant donné la perte de leurs possibilités d'énonciation.

Mots clés: écriture collaborative, récit de fiction, génétique textuelle, discours rapporté, manuscrit scolaire 


\section{The construction of characters' speech during the text production process}

\section{Abstract}

This article describes cases of reported speech (DR) during the production of etiological tales invented by a pair of students (seven years old). Different types of DR will be identified for a series of ten manuscripts, and the genesis of DR within a given writing process will be illustrated from initial formulation to textualization. Based on the theoretical framework of Textual Genetics and the Linguistics of Enunciation, the article examines the procedural dimension of act while writing, observing the role of each student therein. It is noted that the greater number of incidences of DR-113 spoken vs. 29 written-appears to be linked to the complexity of the graphical and enunciative forms of DR in writing, given the loss of enunciative possibilities that they represent.

Keywords: collaborative writing, fiction story, textual genetics, reported speech, school manuscript

\section{La construcción del discurso del personaje en el texto que se está produciendo}

\section{Resumen}

En este artículo se describe el caso del discurso indirecto (DR) durante la producción de cuentos etiológicos inventados en díadas por estudiantes (siete años). Destacaremos los tipos DR de un conjunto de diez manuscritos e ilustraremos la génesis de un proceso de escritura de DR, desde la formulación inicial a su textualización. En el marco teórico de la genética y la lingüística textual de enunciación, observando el rol de cada estudiante, vamos a ver el contexto de la redacción durante su producción. Destacamos que debido a la pérdida de las posibilidades de enunciación del DR escrito, el número de DR por vía oral, 113, por 29 al escrito, parece estar relacionado con la complejidad de las formas gráficas y de enunciación de este ultimo.

Palabras clave: escritura colaborativa, narración ficticia, genética textual, discurso indirecto, manuscrito escolar 


\section{Introduction'}

Ce travail se base sur la genèse et la caractérisation des discours de personnages créés par une dyade d'élèves, lorsqu'ils écrivent ensemble des contes étiologiques inventés. Notre objectif, en premier lieu, est d'identifier et de décrire le discours rapporté (DR) lié aux personnages dans les manuscrits réalisés par les élèves. En second lieu, nous analyserons la genèse du DR dans l'un des processus d'écriture collaboratifs. Nous situons notre étude dans le domaine de la recherche proposée par la génétique textuelle à travers une approche énonciative et dialogique.

La description que nous donnerons à propos du DR dans les productions inventées s'attachera à un point particulier de sa constitution, celui que nous désignons par le "contexte écologique de sa production" (Calil, 2017). Il s'agit de récupérer la construction orale coénonciative du DR, effectuée par le binôme à partir de l'enregistrement vidéo de son processus d'écriture. Ce procédé permet d'analyser et de comparer minutieusement les énoncés écrits avec les énonciations orales en observant sa genèse et sa construction.

À partir de ces conditions, nous avons l'intention de répondre aux questions suivantes: quels éléments linguistiques indiquent la présence de DR? Comment et à quel moment du processus d'écriture les élèves mettent-ils à l'écrit la parole des personnages? De quelle façon se différencie et s'articule le processus énonciatif du DR dans chacun des binômes?

Pour répondre à ces questions, notre analyse se divise en deux parties. 1) analyse quantitative et comparative des DR identifiés dans les dix manuscrits des processus d'écriture et nos propres processus enregistrés en vidéo; 2) analyse microgénétique de DR identifiés dans un manuscrit relatifs à la parole d'un personnage. Dans cet article, nous considérerons la dynamique coénonciative des interlocuteurs en combinaison avec ce qui aura lieu dans le manuscrit, c'est-à-dire que nous analyserons la relation entre la construction du DR (la combinaison orale), son inscription et sa linéarisation dans le manuscrit. Cela permettra de souligner que la transposition de l'oral vers l'écrit engendre une perte des possibilités de représentation du DR.

La pertinence sociale de ce travail vient du dialogue et de la dynamique coénonciative entre les élèves, qui favorise non seulement la réflexion métalinguistique, mais aussi un processus créatif, comme l'a montré Calil dans quelques-uns de ses travaux (Calil, 2012a, 2012b, 2013, 2014, 2016a, 2016b). Notre étude s'intéresse donc à l'intégralité du processus de production du texte, de la réflexion combinée des deux élèves à ce qu'ils planifient en matière d'intrigue pour l'histoire, en plus de l'insertion du DR, de sa linéarisation et de son inscription dans le manuscrit.

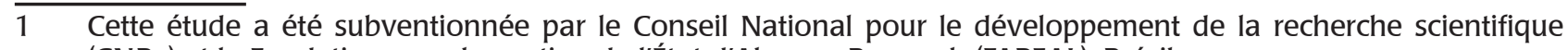
(CNPq) et la Fondation pour le soutien de l'État d'Alagoas Research (FAPEAL), Brésil. 
L'enregistrement vidéo donnant accès à ce qui s'est passé durant la production textuelle permet de mettre en relation ce que les élèves pensaient avant, pendant et après l'écriture du texte. Par le biais de l'enregistrement vidéo, nous pouvons explorer les usages du DR, la façon de créer des élèves et d'insérer les paroles des personnages dans la narration qu'ils sont en train d'inventer. Cela permet d'accéder aux usages et aux connaissances linguistiques ainsi qu'aux commentaires énoncés par les élèves sur le discours rapporté, en visant sa justification et l'explication quant à son écriture sous une forme ou une autre. La reconstitution génétique de l'élaboration de l'histoire par les élèves témoigne de l'écriture comme progression, en la reconnaissant comme un processus lent, long et nécessitant une pratique intense (Kellogg, 2008).

\section{Le discours rapporté comme phénomène énonciatif du dire et de la modalité de la fiction}

Comme affirmé par les travaux de Boré $(2004,2012)$ sur les brouillons scolaires et en accord avec Authier-Revuz (1984), le DR est une marque d'hétérogénéité et fait partie des phénomènes discursifs en relation avec le dialogisme de Voloshinov (2010). Le DR est décrit comme la reprise d'un discours de l'autre; il provient d'une parole prononcée par un tiers et rapportée par le locuteur/énonciateur. Il pose la question cruciale du sens d'un énoncé, d'un événement de parole unique dans une situation particulière d'énonciation. Donc, ce phénomène linguistique est marqué, surtout à l'écrit, par différentes structures grammaticales, des symboles graphiques et de la ponctuation, ainsi que par l'utilisation d'éléments déictiques (comme les pronoms et les adverbes) et de verbes indiquant un acte de parole: dit, raconta, parla, interrogea, cria, chanta, chuchota, pensa, etc. Tous ces éléments linguistiques convergent vers la parole de l'autre et leur grande diversité témoigne de la complexité et de l'hétérogénéité du DR.

La méthode utilisée prendra donc en considération ce phénomène linguisticoénonciatif à partir d'une conception dialogique du langage. Nous considérons comme dialogique l'orientation donnée à un discours en fonction d'autres énoncés (Bakhtin, 1992). Ce mouvement se manifeste à mesure que nous avons des énonciations provenant d'énonciateurs distincts identifiés par d'autres voix, certaines plus présentes, perceptibles et claires, d'autres supposées, déduites ou suspectées. Dans le fonctionnement dialogique du dire, que ce soit dans sa forme "montrée" ou dans sa condition "constitutive", ce qui est dit est toujours fait et effet d'autres dires (Authier-Revus, 1984).

Si nous considérons que les paroles prononcées par le sujet qui parle sont toujours influencées par les paroles des autres, un locuteur élabore nécessairement son discours à partir de celui des autres. C'est ce qu'on appelle le dialogisme. 
Dans le processus de création en temps réel d'histoires inventées, la présence de dialogues ou de paroles de personnages a une valeur dialogique significative. En effet, elle permet d'analyser le dialogisme à partir d'un jeu énonciatif établi entre le scripteur, le narrateur et les personnages. De cette façon, les discussions des élèves au cours de leur processus d'écriture seront considérées comme un moyen d'accéder au fonctionnement dialogique de la construction du DR. La recherche focalisée sur le dialogue des écrivains confirme la nécessité de l'articulation d'une analyse centralisée sur les formulations et les reformulations des apprenants, dans lesquelles le DR se présente selon le point de vue soit du narrateur, soit d'un personnage.

Cette dimension du dialogisme interlocutif, délimité par le dialogue en face à face des locuteurs (Bres, 2005), constitue le point de départ pour dévoiler les relations dialogiques et interdiscursives présentes dans la construction du DR écrit par les élèves. II semble raisonnable de supposer que les élèves, lorsqu'ils écrivent en binôme et se réfèrent au discours de l'autre, mettent en pratique les compétences linguistiques dont ils disposent pour signaler la formation du DR dans sa configuration d'écriture. Les dimensions coénonciative et collaborative sont importantes afin de comprendre comment la formalisation du DR se produit, étant donné que l'on retrouve dans la construction du DR des structures similaires à celles des pratiques discursives du genre textuel de référence.

Nous comprenons cependant que le locuteur, lorsqu'il est narrateur-scripteur, utilisera un certain nombre de dires, issus de divers univers discursifs, en relation avec le contexte culturel et la littératie à l'école qui entoure et fonde sa mémoire comme sujet parlant. Ainsi, nous considérons que l'apparition de DR dans les histoires inventées est liée au principe dialogique. Autrement dit, la construction du DR dépendra des multiples pratiques discursives qui sont nées d'interactions sociales (famille, professeurs, collègues...), ainsi que de l'exposition à la culture littéraire (films, livres de littérature, livres didactiques, bandes dessinées, publicités télévisuelles, musiques...). Comme indiqué dans d'autres travaux (Calil, 2012a; 2012b), les élèves inventent leurs textes à partir de leur univers culturel et des matériels linguistiques qui proviennent de pratiques et de discours auxquels ils sont exposés.

La configuration du produit textuel (manuscrit scolaire) est hautement dépendante de l'immersion du scripteur débutant dans l'univers culturel et littéraire. C'est cette immersion qui va mener "la multiplicité d'éléments linguistiques et discursifs à être réarrangés et combinés dans le manuscrit, pendant le processus d'écriture» (Calil, Amorim et Lira, 2013, p. 77). Lorsque nous décortiquons la construction du DR dans les histoires inventées par les élèves, nous prétendons indiquer comment les relations dialogiques s'opèrent dans leur construction orale et de quelle façon ces relations s'articulent, se présentent ou disparaissent dans la rédaction et la linéarisation des discours rapportés des personnages. 


\subsection{Le discours rapporté dans le récit de fiction}

L'intérêt pour le DR est justifié, de façon générale, par deux aspects: 1) certaines formes de DR (discours direct et discours indirect) font partie du programme d'enseignement; 2) la complexité et l'articulation des aspects linguistiques, sémantiques, discursifs, textuels et graphiques en lien avec les changements au plan énonciatif - surtout ceux qui impliquent le passage de la voix du narrateur à celle du personnage et vice et versa (Bessonnat, 1994; Boré, 2004; Deschildt, 2003; Ferreiro et al., 1996; Plane, Rondelli et Vénérin, 2013).

Deux autres points communs à la grande majorité des travaux sur la forme de représentation du DR dans les brouillons scolaires concernent le choix du matériel textuel qui est étudié. Certains auteurs enquêtent sur les occurrences de DR dans:

1) les produits finis écrits par les élèves (bien que ce soit en différentes versions) (Boré, 2004; 2006; Deschildt, 2003);

2) les produits écrits à partir de textes déjà connus (les élèves réécrivent des textes ou continuent un texte donné avec des personnages prédéfinis ou inventent un récit fictif à partir d'illustrations) (Boré, 2010; Ferreiro et al., 1996).

Parmi ces recherches, on peut citer les études des DR dans les récits de fiction de Boré. Dans ses études, Boré (2006; 2010) a proposé l'identification de trois catégories de DR: "le dialogisme externe", qui met en scène les voix des deux interlocuteurs à partir du locuteur principal; "le dialogue externe représenté, dans lequel les interlocuteurs parlent directement, sans hiérarchie de voix; et "le dialogisme interne représenté", dans lequel celui qui écrit exprime les pensées d'un personnage en se parlant à lui-même ${ }^{2}$. Nous soulignons que la deuxième catégorie est indiquée par l'utilisation d'alinéas.

Les pronoms déictiques et les noms propres, ainsi que les temps du verbe, sont alternés dans l'interlocution sans transition contextuelle d'une voix à l'autre par le narrateur-locuteur. Si, à l'oral, le changement d'intonation met en évidence le discours direct, montrant un jeu de "faire semblant", on le retrouve, dans la représentation écrite, au travers des structures syntaxiques fixes telles que l'occurrence du verbe introducteur, les signes de ponctuation et la parole du personnage. Selon l'auteure, ces traits du dialogisme paraissent être significatifs d'un travail cognitif en cours pour l'élaboration de la fiction.

Dans Ferreiro et al. (1996), l'étude comparative ${ }^{3}$ en trois langues, portant sur la réécriture du conte traditionnel "Le Petit Chaperon rouge", impose un minimum de conditions d'écriture. À titre d'exemple, on compte la présence des personnages (le petit

$\overline{2}$ Boré (2010) a développé dans son ouvrage "Modalités de la fiction dans l'écriture scolaire", plus précisément au quatrième chapitre, les catégories suivantes: "le dialogisme externe", "le dialogue externe représenté» et "le dialogisme interne représenté».

3 L'analyse comparative a été faite sur les textes produits par des enfants de deuxième et troisième année scolaire (CE1-CE2). 
Chaperon rouge, sa grand-mère et le loup), ainsi que l'absence d'éléments traditionnels provenant d'autres contes. Cette étude concerne seulement la relation entre le discours direct (DD) et la ponctuation.

Il est important de noter que le fait que les élèves maîtrisent certains aspects de I'histoire, dans laquelle interagissent toujours deux interlocuteurs, favorise l'apparition du $\mathrm{DD}$, en particulier du fait que les enfants s'appuient sur des structures syntaxiques figées imposées par les consignes. Ce que nous voulons dire, c'est que la réécriture proposée d'un conte traditionnel, basée sur des évènements et situations interactionnelles entre personnages (la mère qui envoie le Chaperon dans la forêt, la rencontre du Chaperon et du loup dans la forêt, la rencontre du Chaperon avec le loup déguisé en grand-mère, etc.), fournit certaines caractéristiques structurelles de l'histoire à reproduire. Les intonations qui découlent des interactions de ces évènements permettent à l'enfant de s'appuyer sur ce qu'il connaît en matière de dialogues et de matérialiser la structure linguistique, dont la trace est explicite par des signes de ponctuation déterminés, comme les points d'interrogation et d'exclamation. Cette proposition et les résultats indiqués ne peuvent pas être généralisés, compte tenu de la spécificité méthodologique de l'étude.

Comme a dit Doquet (2007), se mettre dans la position de celui qui narre pour faire la voix de l'autre - du personnage - implique un questionnement: comment faire parler comme un personnage de conte? Cette question semble être en relation avec le caractère fictif du DR proposé par Boré, dont la présence "réside plutôt dans la position énonciative du sujet de l'écriture" (2010, p. 112). Pour Boré, la capacité fictive a pour condition de se baser sur l'autre. En effet, cela implique l'imagination des actions de l'autre, dans la mesure où il est nécessaire que l'élève mette le "tu» à la place du “je”, le personnage créé et exprimé dans le dialogue externe. Donner une voix à un personnage et délimiter les frontières entre ce dernier et celui qui narre et parle suppose la création d'une instance énonciative. Cette instance ne peut pas également correspondre à la perspective du narrateur.

Nous cherchons à mettre en évidence la construction du DR depuis sa première formulation orale jusqu'à son inscription dans le manuscrit en cours. Les études mentionnées montrent que le $\mathrm{DR}$, quand il est représenté dans le récit écrit, met en évidence les problèmes de changement sur le plan énonciatif ainsi que des rapports entre le DR et les signes de ponctuation. Compte tenu du fait que les actions imaginées par l'autre peuvent donner une marge à l'acte de parole dans un récit, il est important que nous identifiions comment le jeune scripteur construit oralement et représente, dans sa linéarisation, les paroles des personnages dans le récit de fiction inventé. 


\subsection{Inventer et narrer un conte étiologique: du point de vue du narrateur au personnage}

Comme Boré (2010), nous considérons que le DR compris comme reproduction d'un dialogue est un apport important pour la création et pour la construction de récits scolaires. La nécessité de donner une voix au personnage favorise un double jeu énonciatif entre l'enfant qui, comme le narrateur, construit la narration, et le personnage, qui participe au contenu narré (Deschildt, 2003). Cette articulation instaure des dispositifs liés à l'imagination et à la créativité, ainsi qu'à l'univers linguistique et culturel dont les élèves disposent. Cela fait référence, d'une certaine façon, à la fiction comme celle qui imite la réalité, prémisse abordée dans Aristote qui incita Boré (2010) à associer la notion de fiction à la création. Pour elle, l'auteur de fiction se base en réalité sur la matière et, tel l'artisan, fabrique et met en scène les personnages. En ce sens, il est important de considérer l'univers textuel, c'est-à-dire le genre de référence, ainsi que les relations interdiscursives présentes dans l'écriture de l'invention.

L'analyse des études sur la production des récits de fiction fait état du croisement des énoncés caractéristiques de genres textuels divers qui sont tissés et présentés en une seule unité textuelle. Calil $(2008,2013)$ analyse la production libre des récits de fiction présentés par des élèves de six et sept ans, soulignant la présence d'éléments propres aux conventions graphiques utilisés dans les bandes dessinées (onomatopées, métaphores visuelles et types de bulles). La présence de ces éléments est le reflet des innombrables références linguistiques et graphiques qu'on trouve dans les bandes dessinées lues par les élèves à l'époque du recueil de données et démontre, en particulier, la façon singulière qu'a le dialogisme interdiscursif d'interférer dans la construction des dialogues directs représentés dans les récits de fiction.

Choisir et analyser la production textuelle d'histoires inventées comme support matériel pour la représentation de la parole des personnages requiert la prise en compte des plans énonciatifs par lesquels I'histoire est narrée. Le travail de Boré, Calil et Amorim (2015), à partir d'un corpus comparatif de manuscrits brésiliens et français, au-delà de l'identification des formes traditionnelles relatives à la représentation du DR comme le "discours direct" et le "discours indirect", considère le "discours narrativisé» en tant qu'instance énonciative. La proposition d'invention et d'écriture de contes étiologiques, suggérée par les chercheurs, présente un exemple de formes différentes de celles soulignées dans des études précédentes (Boré, 2010).

Le statut du discours narrativisé (DN), différent des structures présentées en discours direct (DD) et discours indirect (DI), qui concernent la syntaxe de l'énoncé, caractérise le mode purement interprétatif, signalant la présence d'un discours autre à l'intérieur du 
discours du narrateur. Ses indices ne sont pas explicites et sont de natures multiples. Par exemple, l'énoncé "ils ont discuté de leurs vacances pendant des heures", classifié dans la recherche de Boré, Calil et Amorim (2015) comme DN, peut nous aider à comprendre cette catégorie d'analyse. Nous signalons que ce lexique verbal ("ont discuté") garde une valeur de "débat". Le complément du lexique verbal présente une situation contextuelle ("de leurs vacances") et une indication de temps ("pendant des heures"), indiquant un examen minutieux au cours duquel sont relevés les "pour et contre" pour "n" sujets, révélés par le référent à la troisième personne du pluriel «ils».

$\mathrm{Si}$, d'un côté, les énoncés ci-dessus comprennent la perspective du narrateur, d'un autre côté, ils présentent l'indication d'un discours autre. Dans ce cas spécifique, nous avons une indication énonciative qui fait référence non seulement à un acte de parole, mais aussi aux changements énonciatifs provenant de cette situation particulière, qui implique un débat du discours entre deux ou plusieurs personnes. Dans cet exemple, nous avons le sujet, c'est-à-dire le thème abordé dans le débat (vacances), mais nous n'avons pas accès au mode d'énonciation qu'il représente. À l'évidence, le DN est une indication d'un événement énonciatif qui fait référence à un acte de langage. Cette catégorie peut présenter plusieurs dimensions extrêmement variables, selon la façon dont le narrateur mobilise et fait référence à l'autre discours.

Plus précisément, nous comprenons le discours narrativisé comme une source dont le narrateur dispose pour souligner l'énonciation du personnage, sans mentionner de quelle manière ses paroles seront représentées. Lors de l'analyse du DN, le scripteur/narrateur présente les faits selon son point de vue, donc selon les possibilités de transposition des énoncés résultant de sa subjectivité.

Dans l'exemple suivant, le DN comporte l'évènement énonciatif à la forme nominale ou infinitive:

a. Elle a demandé la permission de sortir.

b. Elle a demandé à sortir. 
Ces énoncés couvrent l'évènement d'émission de paroles, qui sont résumées et interprétées par ceux qui les énoncent. Voici des variations observées dans les exemples de Jonasson (2003):

(2)

a. Elle a demandé: "Est-ce que je peux sortir?»

b. Elle a demandé si elle pouvait sortir.

(3)

Elle a posé une question.

Comme on peut le voir dans $(2 \mathrm{a}, \mathrm{b})$, il y a une présentation de la parole de "elle" introduite par le verbe dicendi "demander» et cette parole est respectivement rapportée sous la forme DI et DD. Cependant, la présentation de l'énoncé (3) a une forme encore plus réduite que celle de l'énoncé (1). "Elle a posé une question" n'exprime pas de remise en cause; sa valeur illocutoire de "dire" indique un type d'acte énonciatif sans préciser son contenu. Ceci est un exemple de DN réduit à la plus simple forme de description d'événement.

Bien que les élèves ne maîtrisent pas les structures linguistiques complexes et sophistiquées de représentation écrite du DR, cet aperçu suggère la richesse de sa construction chez les élèves au début de l'école primaire. Mais le fait que ces analyses ont pour objet d'étude du produit textuel ne nous aide pas à comprendre comment le DR a été construit pendant la production du texte, ou les éléments linguistiques qui étaient en concurrence au cours de leur textualisation.

Notre travail cherche donc à comprendre le phénomène linguistique, non pas à partir du produit textuel, mais à son état naissant et procédural. De plus, la nature logique de notre étude est essentielle pour l'analyse du phénomène linguistique tel qu'il émerge puisque nous nous intéressons à l'invention de contes, sans orientation didactique spécifique quant au positionnement énonciatif pour le développement de l'histoire du type "narrer à la première ou troisième personne" comme le font Boré (2006) et Ferreiro et al. (1996).

\section{Méthodologie}

Le corpus a été rassemblé en 2012 dans une école de la ville de Maceió (Brésil), auprès d'élèves âgés de six à sept ans issus de milieux socio-économiques similaires, et ayant accès à la culture écrite véhiculée par la littérature pour enfants, les films, les émissions de télévision, les jeux électroniques, etc. La situation d'écriture a été effectuée 
à partir du projet didactique "Contes du comment et pourquoi", mis en place dans une classe de deuxième année de l'école élémentaire.

Le projet fut conduit par l'enseignante elle-même, durant trois mois, et faisait partie des activités d'enseignement de la langue portugaise. Environ 30 contes étiologiques ont été lus et dix propositions de production textuelle ont été faites aux élèves. Plus précisément, dans chaque proposition de production textuelle, les élèves, en équipe de deux, devaient se mettre d'accord et inventer un conte. Après s'être mis d'accord, ils recevaient un papier et un stylo pour procéder à l'écriture. Nous avons fait un enregistrement vidéo de tout le processus d'écriture d'un seul binôme. Pour préserver l'environnement didactique, nous avons évité d'interférer dans le processus interactionnel entre élèves et professeure. La caméra a été montée sur un trépied et l'équipe technique et les chercheurs se retiraient de la salle de classe.

Cette posture méthodologique conserve un caractère ethnolinguistique et permet, à partir d'un enregistrement vidéo, d'apprendre la complexité et la richesse des facteurs didactiques, pragmatiques, linguistiques et anthropologiques qu'impliquent les situations effectives de production d'un texte dans la salle de classe. En plus de préserver le temps et l'espace qui sous-tendent la construction du texte ${ }^{4}$, ce qui distingue méthodologiquement notre travail de ceux cités ci-dessus est que les élèves ont été placés en situation d'écriture collaborative, favorisant ainsi le dialogue entre les pairs et la verbalisation de ce que pense celui qui écrit, au moment précis pendant lequel le binôme planifie ce que sera la composition du texte.

\subsection{Procédures analytiques}

Un facteur essentiel pour l'analyse quantitative du DR est l'identification de sa présence dans les manuscrits produits. Pour cela, nous identifions les marques de "personne" dans le discours (pronoms personnels et possessifs), les verbes de parole ou expressifs (dicendi, sentiendi) et le contexte spatio-temporel lié aux personnages. Le système de ponctuation, les changements de ligne et les marques graphiques ont aussi été pris en considération, ainsi que les éléments sémantiques et lexicaux relatifs aux DR. Ces aspects, qui concernent l'insertion de personnages dans le récit inventé, "peuvent toucher des destinataires différents, en effet, elles s'adressent fictivement à un personnage et narrativement au lecteur" (Deschildt, 2003, p. 144). Les discours ne sont pas juste cités par un narrateur. Comme proposé par Boré (2012), le DR et particulièrement les paroles des personnages occupent un point crucial dans la création du récit. L'identification de ces éléments a permis la catégorisation des formes suivantes de DR: DD, DI et DN.

$4 \quad$ Il est important de noter que les textes produits sont désignés comme manuscrits scolaires, une fois qu'on les considère comme produits d'un processus d'écriture réalisé dans le cadre d'une demande scolaire (Calil, 2008). 
L'indice linguistique du DN, au contraire du DI et du DD, n'assume pas une forme syntaxique fixe. Le DN se révèle à partir d'une multitude de formes qui sont textuellement disposées dans l'énoncé continu. Ce type de DR "demande une attention différente aux verbes de parole qui peuvent s'actualiser par des lexèmes, non pas de reprise du verbe 'dire', dominant dans la version en discours direct, mais de reformulation du contenu même des paroles de personnage" (Plane, Rondelli et Vénérin, 2013, p. 221). Autrement dit, le DN est compris comme catégorie purement interprétative, compte tenu du fait qu'il mobilise dans son énoncé des verbes de parole et des lexiques qui indiquent un événement énonciatif.

Les catégories d'analyse dans le processus oral, extrait de la combinaison orale, suivent l'identification des mêmes éléments syntaxiques. Ainsi, nous leur associons des marques segmentaires et suprasegmentaires. En partant des caractéristiques du DR à l'oral décrites par Rosier (1999), nous délimitons la frontière entre le discours reportant et reporté, considérant les marques d'énonciation, les pauses et les modulations d'intonation qui segmentent l'énoncé. L'insertion de ces éléments, combinés au verbe, à la voix et à la gestuelle, est un indice important pour l'identification de la représentation du DR à l'oral.

\section{Présentation et analyse des résultats}

\subsection{Vue descriptive du discours rapporté dans les produits (manuscrits) et ses processus}

Nous pouvons identifier 142 énoncés oraux et écrits liés au DR dans la genèse textuelle des dix contes étiologiques inventés. Il y a certainement une relation entre le DR construit oralement et celui qui fut graphiquement inscrit. Parfois, un DR est inscrit après un long processus de formulation et de reformulation orale. À d'autres moments, un DR est écrit tel qu'il est proposé oralement. Néanmoins, la différence entre les quantités de DR formulés oralement et le DR effectif par écrit est notable, comme le montre le graphique ci-dessous.

Graphique 1 - Le discours rapporté dans les dix contes inventés (Processus et Produits)

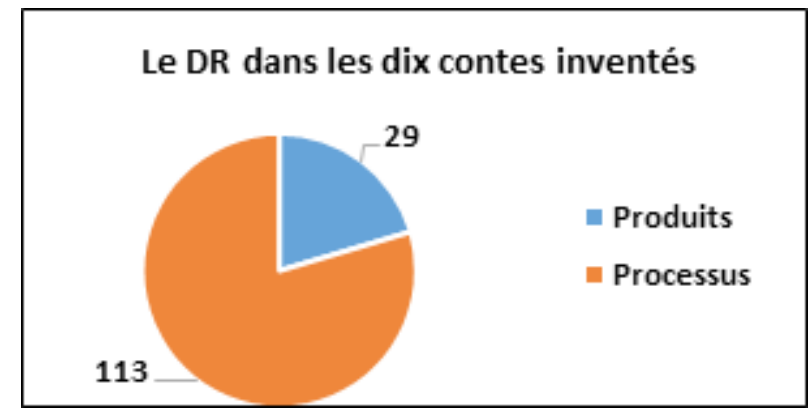


Dans les processus, 113 cas (80 \%) de DR furent constatés, alors que 29 cas (20 \%) furent constatés dans les produits. Cette différence indique une proportion d'environ quatre DR oraux pour un DR écrit, ce qui suggère que la présence d'un DR dans une histoire inventée est le résultat d'une genèse énonciative intense.

La comparaison entre les types de DR, tant à l'oral (processus) qu'à l'écrit (produits) dans chacune, apporte un autre aspect intéressant.

Graphique 2 - Types de discours rapportés (Processus et Produits)

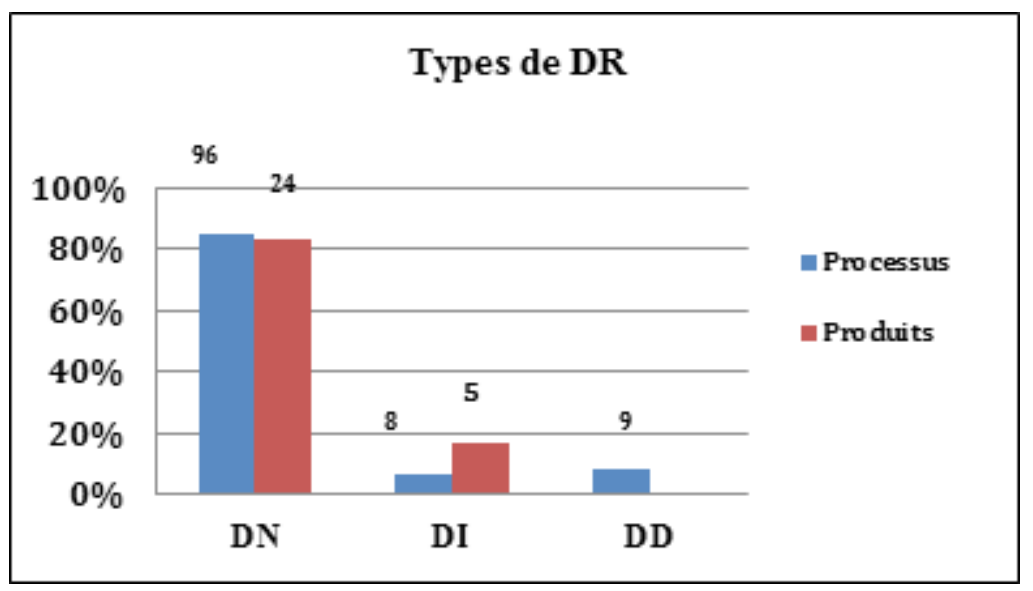

Proportionnellement, la différence accentuée entre les cas de DR oraux et de DR écrits ne se manifeste pas par rapport aux types de DR. Dans la totalité des types de DR, I'un comme l'autre prédomine dans la quantité de cas de DN (85\% pour le registre oral et $83 \%$ pour le registre écrit).

Les nombreuses occurrences de DN dans ce corpus se traduisent en différentes formes en regard à l'étude comparative de manuscrits français et brésiliens réalisée par Boré et al. (2015). Alors que notre recherche se focalise sur les structures linguistiques représentées dans les productions textuelles de deux élèves, la description par Boré, Calil et Amorim (2015) des marques de DR dans les manuscrits scolaires des élèves brésiliens et français âgés de sept ans montre que la forme prépondérante est le DD. En ce qui concerne leurs manifestations écrites, le nombre d'occurrences de DD et de DI diverge considérablement. On observe $17 \%$ dans la forme DI (cinq cas) alors que I'on ne retrouve aucun cas de DD sous la forme écrite.

Quant à l'émergence de ces types de DR au cours du processus d'écriture, à savoir sa représentation orale au cours de l'écriture, il est nécessaire de souligner l'importance de l'extrait suprasegmentaire comme élément différenciateur. Les élèves, en insérant la parole des personnages dans les contes inventés, posent des gestes, des mouvements et s'expriment comme s'ils étaient le personnage. En prenant en compte cet aspect comme 
marqueur de DD à l'oral, nous pouvons dire qu'il y a eu neuf cas de DD (8 \%), contre huit cas de DI (7 \%).

\subsection{La construction de DR dans un processus d'écriture}

Bien qu'il y ait des différences quant à la fréquence des cas par catégorie, les types de cas de DR, donc DI, DD, DN, dans les manuscrits, coïncident avec ce qui a déjà été trouvé dans les études de Boré (2004) et Boré, Calil et Amorim (2015). Cependant, la manière dont les formes de DR sont construites avant de trouver leurs configurations écrites est très peu étudiée, étant donné que les notes laissées sur le papier, que ce soit celles du brouillon ou de la version finale du produit textuel, ne prennent pas en compte ce qui se passe au moment de l'inscription ${ }^{5}$

L'analyse que nous proposons de la genèse du DR identifie et décrit comment ce dernier a été construit oralement jusqu'au moment où il a pris une forme écrite. Dans cette section, nous montrerons la différence entre les formes énonciatives orales des DR proposés par les élèves et nous analyserons ensuite la genèse et la construction du DR au moment de l'inscription et de la linéarisation du conte étiologique.

Entre Caio et Igor, il y a des différences d'importance relatives à la quantité de DR proposés.

Graphique 3- Le discours rapporté par les élèves (Processus)

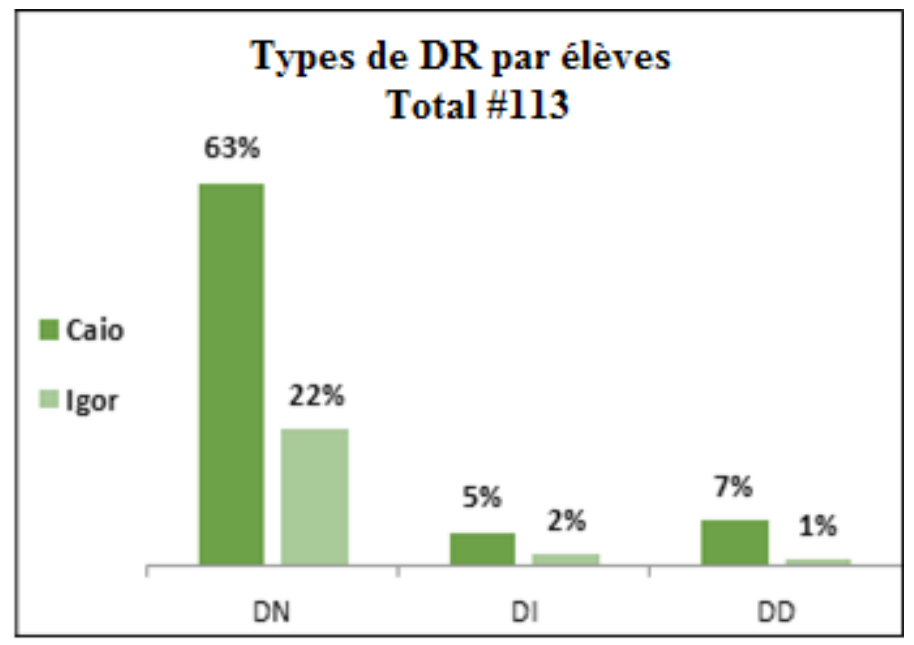

Dans tous les processus, on dénote trois types de DR (113), répartis en 96 DN, 8 DI et 9 DD, parmi eux 75 \% (85) furent élaborés par Caio, répartis en 71 DN, 6 DI et 8 DD et $25 \%$ (28) par Igor, répartis en 25 DN, 2 DI et 1 DD.

$5 \quad$ Bien que les marques laissées sur le papier peuvent donner des indices d'une version à l'autre, et permettent de récupérer chaque altération, ce qui a été écrit ne montre pas le moment réel de l'écriture ou la façon pensée par l'élève pour écrire le DR, avant de l'inscrire sur la feuille de papier. 
Ces résultats révèlent deux aspects du processus coénonciatif établi pendant le moment de la "combinaison" et de «l'inscription-linéarisation" des récits inventés par les élèves:

1) Aucun DR repéré dans les manuscrits ne fut écrit sans formulation et reformulation orale. La question est de savoir comment s'est déroulée cette construction et quels types d'altérations ont eu lieu.

2) Les faits montrent que Caio a plus contribué à la construction de ces DR. Cette différence suggère une habileté linguistique et textuelle majeure de Caio pour la construction du DR.

Pour l'analyse du processus de construction du DR, nous choisissons le processus d'écriture du manuscrit "Por que existe o arco-íris" (Pourquoi l'arc-en-ciel existe-t-il?). Nous sélectionnons ce processus parce qu'il illustre la dynamique coénonciative intense, le rôle de chaque élève dans sa construction et sa relation avec le DR et la construction du personnage de cette histoire inventée.

Figure $1^{6}$ - État du manuscrit scolaire "Por que existe o arco-íris» (Pourquoi l'arc-enciel existe-t-il?), lignes 8 à 13, linéarisé entre 43':30" et 59':06" (sixième processus, $07 / 05 / 2012)$

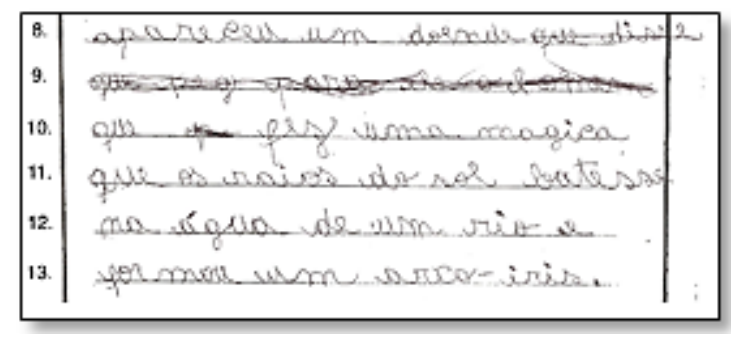

Dans ce sixième conte étiologique inventé par le binôme, Igor était responsable de la rédaction de I'histoire et Caio de la dicter. Il y a une longue rature entre les lignes huit et neuf, qui commence juste après "apparaît un elfe / apareceu um duende», sous laquelle il est possible d'identifier le registre "qui dit / que disse» (ligne huit) et "qui prit pour lui l'homme / que peg para ele o homem" (ligne neuf) . Cependant, l'enregistrement vidéo montre que la rature n'a pas été faite en une seule fois. Elle n'impliquait pas seulement un problème textuel, par exemple le problème orthographique lié à l'orthographe du mot "prendre / pegar" de la ligne neuf, mais aussi d'autres problèmes, ce que nous examinerons. Malgré

$6 \quad$ Le texte en portugais est reproduit avec l'orthographe, la syntaxe et la ponctuation originale. La traduction en français est littérale:

8. apareceu um doende que disse (apparut un elfe qui dit)

9. que peg para ele o homem (qui pripour luil'homme)

10. que $p$ fez uma magica (qui $p$ fit un tour de magie)

11. que os raios do sol batesse (que les rayons du soleil frappait (frappaient))

12. na água de um rio e (dans l'eau de la rivière. et)

13. formou um arco-iris. (se formait un arc-en-ciel)

7 Pour la traduction en français, nous avons choisi de maintenir la construction syntaxique portugaise (brésilienne). 
le fait que ces ratures aient l'air d'avoir été faites en continu, en barrant d'un trait toute la phrase "qui dit (ligne huit) qui prit pour lui l'homme (ligne neuf) / que disse (ligne huit) que peg para ele o homem» (ligne neuf), elles ont été faites à des moments différents et pour des raisons distinctes, dont des problèmes orthographiques et syntaxiques, mais surtout, de représentation du discours rapporté.

C'est justement ce problème qu'il est intéressant d'analyser. Nous pouvons observer que la rature à la suite de "apparu un elfe / apareceu um duende" indique la linéarisation d'un discours rapporté par le narrateur du personnage de l'elfe: "Apparu un elfe qui lui dit I'homme / apareceu um duende que disse para (ele) o homem".

Cet énoncé écrit porte les marques syntaxiques et sémantiques de la construction du DR du "duende" (elfe) dans sa forme indirecte, à savoir le fait que le scripteur-narrateur s'appuie sur la structure " $X$ dit que $Y$ ". La rature efface une tentative de construction de DR. Depuis la position énonciative du narrateur, on peut interpréter ce qu'il y a sous la rature par deux tentatives de formulation du DR: "un elfe dit que / um duende disse que" et "un elfe lui (I'homme) dit / um duende disse para ele". La présence du verbe "dire", à la troisième personne du passé composé, suivie de la conjonction "que", de la préposition "pour / para" (pour) et du nom du personnage (l'homme) constitue l'introduction du DR. Cependant, elle indique aussi une hésitation ou une difficulté à articuler l'objet direct (“dit que / disse que") et l'objet indirect ("dit à / disse para") relatifs à sa construction. Le fait que l'enfant ait raturé semble donc être lié à la construction du DR.

Tout de suite après cette rature, les lignes 10, 11, 12 et 13 furent écrites:

qui fit un tour de magie (ligne 10)

que les rayons du soleil frappait (frappaient) (ligne 11)

dans l'eau d'une rivière et (ligne 12)

se formait un arc-en-ciel (ligne 13).

Dans ces lignes, au contraire de ce qui fut identifié sous les ratures, il n'y a pas de cas de DR clairement délimité. Du point de vue textuel, ce qui fut écrit dans les lignes 11, 12 et 13 est lié sémantiquement à "[l'elfe] fit un tour de magie / [o duende] fez uma mágica" (ligne 10). C'est-à-dire que le tour de magie fait par l'elfe fit en sorte que "les rayons du soleil frappait(ent) dans l'eau d'une rivière / os raios de sol batesse(m) na água de um rio» et eu comme effet ou pour résultat "la formation (I'apparition) de l'arc-en-ciel / a formação (aparecimento) do arco-íris".

Dans ce texte de corédaction, l'expression verbale efit un tour de magie / fez uma mágica" correspond au verbe de parole, indiquant, du point de vue de la construction syntaxique énonciative, la présence du DR. L'acte de "faire le tour de magie" serait lié à 
l'acte d'énoncer ou de lancer un sort, en disant des paroles magiques pour que quelque chose se réalise. Les paroles magiques dites par l'elfe étaient "que les rayons du soleil frappait(ent) dans l'eau de la rivière / que os raios de sol batesse(m) na água de um rio". Ceci peut être plus facilement observé si l'on substitue le "fit un tour de magie / fez uma mágica» par des constructions impératives comme “j'ordonne» ou “je veux»: [J’ordonne/ veux/désire] que les rayons du soleil frappent dans l'eau d'un fleuve.

L'énoncé écrit semble soutenir la cooccurrence de deux possibilités d'énonciations du scripteur/narrateur, similairesà cellequi setrouvait sousla rature précédente. Premièrement, "faire un tour de magie" pour qu'apparaisse l'arc-en-ciel. Le fait que le narrateur décrive quel fut le tour de magie réalisé par l'elfe et la forme verbale en Portugais "batessem" soit "frappent" (troisième personne du subjonctif imparfait) l'indique. Deuxièmement, "dire le tour de magie", à savoir l'elfe qui énonce les paroles de l'enchantement pour que l'arc-en-ciel apparaisse et la linéarisation "que les rayons du soleil» le suggèrent. Cette ambiguïté énonciative entre "décrire" le tour de magie et "dire" le tour de magie faite par l'elfe s'assimile à ce que les autres études sur les caractéristiques du DR avec les jeunes scripteurs débutants suggèrent. La construction du DR dans sa forme écrite exige du scripteur une maîtrise et l'articulation de différents niveaux linguistiques et énonciatifs.

Cependant, la présence du DR dans le manuscrit ne permet pas de savoir comment ce DR fut construit. L'analyse du dialogue entre les élèves apporte des éléments énonciatifs importants en relation à la rature et à la présence de marques orales dans la construction de la parole du personnage:

Texte dialogal (TD): sixième processus (manuscrit "Pourquoi l'arc-en-ciel existe-t-il») $50^{\prime} 34^{\prime \prime}-51^{\prime} 54^{\prime \prime}\left(1^{\prime} 20^{\prime \prime}\right)^{8}$

981. CAIO: (En lisant [apparut un elfe]) Ainsi, le... le... I'elfe: Apparut un elfe... que l'emmena à... qui fit un tour de magie; (II fait les gestes avec les bras comme si la pluie était en train de tomber). Chaque fois qu'il pleuvait... et apparaissait le soleil... apparaissait le soleil... toujours apparaitrait un arc-en-ciel. Ça va?

982. IGOR: (II pense) II (Brève pause) II...

983. CAIO: (Il change d'intonation comme s'il était le personnage.) Chaque fois que... il pleut... (II reparle normalement) et que l'elfe apparait et fait un tour de magie (II gesticule de nouveau avec les bras et les mains, faisant désormais les gestes d'un tour de magie) et... que chaque fois qu'il pleuvait... et... et le soleil frappait dans l'eau... (II fait un ton de suspense au moment de dire "apparaittrait») ... apparaîtrait...

984. IGOR: (II parle à la suite.) L'arc-en-ciel!

$8 \quad$ Cet extrait concerne la session d'écriture dans laquelle se déroule la construction de ce DR. Le guillemet simple représente les minutes et le guillemet double, les secondes. Le temps total d'analyse correspond à une minute et vingt secondes (1'20"). 
985. CAIO: Voilà. On fait ça? Le, l'elfe. Maintenant il va falloir supdisparaître tout ça. (Brève pause)D'íci (II montre le trait Iqui dit que pri pour lui I'homme]) II faut qu'il disparaisse parce que lui il va rien dire.

986. IGOR: Oui. Ça ici (II montre [qui dit]) il va falloir que ça disparaisse? (En faisant une rature sur "qui dit" [qui dit]).

987. CAIO: Oui. Là. sup-supprime (Montrant la ligne huit, Iqui prique peg para ete o homem e pour lui I'homme])

988. IGOR: C'est pas supprimer (En rayant [qui pri pour lui I'homme])

989. CAIO: C'est supprimé. (Igor continue de raturer, faisant plusieurs ratures à la ligne huit)

990. IGOR: C'est pas supprimer. C'est rayer.

991. CAIO: Ah ok. Rayer.

L'enregistrement vidéo permet d'observer simultanément ce qu'ils disent, ce qu'ils écrivent et les gestes et expressions faciales qui accompagnent chaque action. Dans ce texte dialogal, nous avons transcrit ce qu'ils ont dit lorsqu'ils ont raturé "qui dit (ligne huit) que pri pour lui l'homme (ligne neuf) / que disse (ligne huit) que peg para ele o homem (ligne neuf)". La rature s'est produite durant les tours 986 et 988.

En premier lieu, au tour 981, Caio associe le personnage "elfe" à l'acte de "faire un tour de magie". Ensuite, au même tour, Caio change le ton de sa voix, en gesticulant et en faisant des pantomimes comme s'il était lui-même le personnage en disant ce qu'il va se passer pour que l'arc-en-ciel apparaisse. Caio introduit un DI, rapportant ce qu'aurait dit l'elfe quant au tour de magie: "Chaque fois qu'il pleuvait et le soleil apparaissait... Apparaissant toujours un arc-en-ciel. Ça va? / Toda vez que chovesse e aparecesse o sol... sempre apareceria um arco-íris. Que tal?».

Avec l'introduction du personnage elfe dans l'histoire et du tour de magie qu'il fait pour faire apparaître l'arc-en-ciel, Caio fait des signes suprasegmentaux (changement de voix, intonation, rythme, pause et mélodie) et gestuels (pantomimes avec les bras, imitation du sort qu'il lance et de la pluie tombante). Ces points, et tout particulièrement le changement d'intonation, indiquent une représentation orale du DD, commun dans les situations de récits oraux. Cependant, la construction linguistique de l'énoncé de Caio qui réfère au DR du personnage a les propriétés d'un discours rapporté indirect (DI), à savoir le fait qu'il rapporte entre les tours 981 et 983 ce que dit l'elfe "Apparu un elfe qui 'fit un tour de magie' [il dit] 'que chaque fois qu'il pleut et qu'apparaît le soleil apparaîtrait toujours un arc-en-ciel' / apareceu um duende que 'fez uma mágica' [ele disse] 'que toda vez que chovesse e aparecesse o sol sempre apareceria um arco-íris'». 
Cette imbrication entre les formes DD et DI peut être plus facilement observée à partir de sa séparation:

\begin{tabular}{|l|l|}
\hline Forme DD & $\begin{array}{l}\text { Apparu un elfe qui fit un tour de magie et dit: } \\
\text { - Chaque fois qu'il pleut et apparaît/frappe le soleil, apparaîtra } \\
\text { toujours un arc-en-ciel. }\end{array}$ \\
\hline Forme DI & $\begin{array}{l}\text { Apparu un elfe qui fit un tour de magie et qui dit que chaque fois } \\
\text { qu'il pleuvrait et apparaîtrait/frapperait le soleil, apparaîtrait tou- } \\
\text { jours un arc-en-ciel. }\end{array}$ \\
\hline
\end{tabular}

La formulation orale du DR fait par Caio a résolu l'impasse dans laquelle il se trouvait quant à l'introduction du DR qui avait déjà été écrit, provoquant, entre les tours 986 et 988, la rature faite par Igor sur: "que dit / que pri pour lui I'homme / que disse / que peg para ele o homem". Le dialogue ci-dessus montre justement la relation entre le DR et la rature. En même temps, il indique le passage du DR oral proposé par Caio vers sa forme écrite dans laquelle il manque encore certains détails. Le manuscrit préserve seulement la première partie du DR oral. C'est-à-dire que l'introduction du tour de magie faite par le personnage et ce qu'il dit "qui fit un tour de magie que chaque fois qu'il pleut et qu'apparaît/frappe le soleil / que fez uma mágica que toda vez que chovesse e aparecesse/ batesse o sol" sont linéarisés comme "qui fit un tour de magie que les rayons du soleil frappent dans l'eau d'une rivière / que fez uma mágica que os raios de sol batesse(m) na água de um rio» (lignes 10, 11 et 12).

La linéarisation du DR oral a provoqué une réduction et un changement du discours du personnage. Les lignes 10, 11, 12 présentent une forme écrite de $D R$, avec des marques de DD et DN. Bien que les éléments de base du DD du personnage aient été conservés, ce qui est indiqué par le "que / que" et par le "frappent / batesse" dans l'énoncé "que les rayons du soleil frappent dans l'eau d'une rivière", sa linéarisation décrit la magie faite («les rayons du soleil frapperont dans l'eau d'une rivière / os raios de sol bateram na água de um rio"), suggérant une narrativisation par l'elfe.

La seconde partie du DR oral («apparaîtrait toujours un arc-en-ciel / sempre apareceria $o$ arco-íris") cesse d'avoir une valeur de DR dans le manuscrit, en devenant une partie des événements narrés et résultant de l'effet du tour de magie lancé: «et se forma l'arc-en-ciel / e formou (-se) o arco-íris".

Dans cette conception dialogique du langage, l'occurrence du DR souligne les relations discursives et interdiscursives présentes dans la mémoire de l'élève comme modèle pour sa rédaction. Si l'on considère que Caio, lorsqu'il énonce le DR, se met dans la position de celui qui narre pour imiter la voix du personnage, on observe une situation de dialogisme. Pour constituer son discours, un locuteur se base nécessairement sur d'autres discours. Autrement dit, l'exposition à l'alphabétisation, à partir de l'immersion dans l'écriture 
du genre textuel de référence, permet la mobilisation de structures similaires à celles rencontrées dans les pratiques discursives auxquelles les élèves sont exposés.

L'énoncé "J'ai des pouvoirs magiques, claque des doigts et, paf, la nourriture apparaît! / Eu tenho poderes mágicos, basta estalar os dedos e, ploft, a comida aparece!» (Zatz et Abreu, 2010, p. 19), présent dans les contes lus, montre la relation interdiscursive avec le DR créé par Caio. L'influence de cet énoncé est évidente dans la représentation du discours de l'elfe et démontre que les élèves sont conditionnés à utiliser le matériel linguistique dont ils disposent. L'action de "faire un tour de magie", exprimée par les pantomimes de Caio, est l'effet du discours d'un autre et constitue une marque interdiscusive donnée par les références culturelles. Nous pouvons déduire que la textualisation de ce qu'énoncent les personnages passe par le discours de l'autre, mais sa configuration indique un ordre particulier délimité par d'autres facteurs, comme l'utilisation inadéquate des démarcations du discours de l'autre - absence de points de ponctuation, ou encore absence d'alinéas -, pendant la linéarisation.

\section{Conclusion}

Dans nos résultats, nous avons identifié une différence remarquable entre le nombre de cas de DR dans les processus et dans les produits. L'analyse du processus d'écriture du conte "Pourquoi les arcs-en-ciel existent-t-ils? / Por que existe o arco-íris?" suggère que la condition coénonciative en est responsable. Cette condition a joué un rôle crucial dans la genèse et la construction du DR. L'alternance des tours, les désaccords et les hésitations, les gestes et expressions faciales et, surtout, la nécessité fictionnelle d'inventer des personnages ont favorisé la production de DR. En outre, ces aspects ont mis en évidence I'articulation des formes orales du DR et son passage vers la forme écrite.

En fait, les élèves sont en train de maîtriser les formes orales du DR et son passage vers la forme écrite, ce qui est révélé par la présence de cette forme DD dans les processus et de son absence dans les écrits produits. Dans le processus analysé, nous avons pu observer comment Caio imite l'intonation de voix du personnage lorsqu'il fait le tour de magie pour qu'apparaisse l'arc-en-ciel. Il est utile de souligner que l'absence de DD dans les manuscrits peut être mise en relation avec le manque de connaissances des élèves au sujet du fonctionnement et de l'utilisation des signes de ponctuation. En plus d'être pratiquement absents dans les manuscrits, dans l'ensemble des processus d'écriture de ce binôme, il n'y a aucune discussion sur ces signes de ponctuation. À aucun moment les élèves ne proposent de ponctuer ou de marquer graphiquement les paroles d'un personnage. Cet aspect mériterait d'être approfondi, mais peut probablement être l'une des raisons qui expliquent la grande quantité de DN dans les manuscrits. 
Comme nous l'avons vu dans le dialogue analysé, l'imbrication du DD et du DI dans leur forme orale suggère que la transformation du DN est le résultat de sa linéarisation et du manque de maîtrise du système de ponctuation.

La textualisation du DR par ces élèves, depuis sa formulation et reformulation orale jusqu'à sa linéarisation, dépendrait au moins de trois facteurs:

1) De la complexité du système de représentation écrite du DR et des connaissances linguistiques qu'ont les élèves.

2) De l'articulation entre la formulation orale coénonciative et de sa linéarisation et son articulation comme manuscrit en cours de production.

3) Des propriétés du genre textuel imposé.

Ces facteurs, associés au savoir littéraire des élèves, - tel qu'indiqué par les paroles de Caio lors de l'introduction du tour de magie fait par l'elfe, récupérant les structures linguistiques communes aux récits de fiction dans lesquels il y a des ensorcellements et des enchantements - seraient interdépendants et favoriseraient l'apprentissage et la maîtrise des formes de représentation de DR par les élèves.

Les implications didactiques correspondant à l'acte d'écrire de façon collaborative favorisent le dialogue interlocutif ainsi que la construction coénonciative du texte en cours de production. Cette forme d'interaction verbale met en évidence les connaissances linguistiques et textuelles des apprenants, car les élèves doivent les utiliser comme arguments pour l'entrée de plusieurs éléments à linéariser lors de l'écriture. En ce qui concerne la linéarisation du discours rapporté, nous avons noté que le caractère "explicatif» du conte étiologique peut avoir influencé le nombre d'occurrences de DN.

La difficulté d'insertion du DR dans le texte en cours de production suggère peut-être la nécessité d'un travail plus systématique, du point de vue didactique, qui mettrait l'accent sur le fonctionnement de cet élément linguistique. L'écriture avec la focalisation sur les interférences didactiques, telles que proposées par les recherches sur les méthodes de séquence d'enseignement (Bronckart, 1997), peut ajouter aux pratiques de textualisation une approche plus régulière et un apprentissage systématique, fournissant un suivi plus réflexif de l'utilisation que l'écrivant fait de la langue. En effet, l'intervention de l'enseignant lors de la formulation/inscription, surtout à l'étape de révision, au cours de laquelle les élèves lisent la production, serait intéressante puisqu'il s'agit de moments particulièrement opportuns pour observer ce qui a été développé par les élèves. Par exemple, l'enseignant pourrait leur demander, au sujet du mode d'entrée de DR, quelles sont les marques d'ouverture et de fermeture des dires, dans le but d'obtenir une explication quant à son 
écriture. Cela offrirait aux élèves la possibilité d'engager une réflexion concernant le discours du personnage.

Une lecture attentive de notre travail peut guider l'enseignant de la langue maternelle vers un enseignement systématique de la production écrite axée de façon plus catégorique sur le genre, mais aussi sur l'utilisation d'éléments lexicaux et grammaticaux, tout en maintenant leur pratique d'enseignement quant à la collaboration et à la production écrite comme processus d'écriture.

\section{Références}

Authier-Revuz, J. (1984). Hétérogénéité(s) énonciative(s). Langages, 19(73), 98-111.

Bakhtin, M. (1992). Estética da criação verbal. São Paulo: Martins Fontes.

Bessonnat, D. (1994). Parole des personnages: bâtir une progression. Pratiques, 83, 5-34.

Boré, C. (2012). Le discours direct dans des écrits fictionnels scolaires, marques et signification. In S. Branca-Rossof et al. (dir), L'hétérogène à I'œuvre dans la langue et les discours (p. 117-134). Limoges: Lambert-Lucas.

Boré, C. (2010). Modalités de la fiction dans l'écriture scolaire. Paris: L’Harmattan.

Boré, C. (2006). L'écriture scolaire de fiction comme rencontre du langage de l'autre. Repères, $33,37-60$.

Boré, C. (2004). Discours rapportés dans les brouillons d'élèves: vrai dialogisme pour une polyphonie à construire. Pratiques, 123, 143-169.

Boré, C., Calil, E. et Amorim, K. L. (2015). Étude comparative des formes de discours rapportés dans des contes des origines chez des scripteurs français et brésiliens (7-8 ans): premiers résultats. Document téléaccessible à l'adresse <http://www.shs-conferences.org/articles/shsconf/pdf/2015/03/ shsconf_iaimte2013_02001.pdf>

Bres, J. (2005). Savoir de quoi on parle: dialogue, dialogal, dialogique. In J. Bres et al. (dir.), Dialogisme, polyphonie: approches linguistiques (p. 47-61). Paris: De Boeck.duculot.

Bronckart, J.-P. (1997). Activité langagière, textes et discours. Paris: Delachaux et Niestlé.

Calil, E. (2017). Rasura oral comentada: definição, funcionamento e tipos em processos de escritura a dois. In C. L. Da Silva, A. Del Ré et M. Cavalcante (dir.), A criança na/com a linguagem: saberes em contraponto (p. 161-192). Porto Alegre: UFRGS.

Calil, E. (2016a). The meaning of words and how they relate to the ongoing text: A study of semantic comments made by two 7-year-old schoolchildren. In M.-F. Morin, D. Alamargot et C. Gonçalves. Perspectives actuelles sur l'apprentissage de la lecture et de l'écriture / Contributions about learning to read and write (p. 195-220). Sherbrooke: Éditions de l'Université de Sherbrooke.

Calil. E. (2016b). Writing, memory and association: newly literate students and their poetry creation processes. Recherches Textuelles, 13, 105-121.

Calil, E. (2014). Séries associatives à l'oeuvre dans l'écriture d'un poème en salle de classe: de «jaune» à "avec la foi». In M. Jacques et C. Raulet-Marcel (dir.), Inventions de l'écriture. Dijon: Éditions Universitaires de Dijon.

Calil, E. (2013). Dialogisme, hasard et rature orale Analyse génétique de la création d'un texte 
par des élèves de 6 ans. In E. Calil et C. Boré. (dir.), L'école, l'écriture et la création: Études franco-brésiliennes (p. 157-188). Louvain-la-neuve: L'Harmattan-Academia.

Calil, E. (2012a). The Gluttonous Queen: dialogism and memory in elementary school writing. Bakhtiniana: Revista de Estudos do Discurso, 7, 24-45.

Calil, E. (2012b). Dialogues between two pupils during the process of writing a fictional story Verbal erasures and their forms of representation. In F. Cooren et A. Létourneau (dir.), (Re) presentations and Dialogue (p. 325-341). Amsterdam: John Benjamins.

Calil, E. (2008). Cadernos de histórias: O que se repete em manuscritos de uma criança de 6 anos? In V. S. Blas. (dir.), Mis primeros pasos. alfabetización, escuela y usos cotidianos de la escritura (p. 55-70). Gijón: TREA.

Calil, E., Amorim, K. et Lira, L. (2013). Letramento e processo de escritura de alunos recémalfabetizados. Caderno Cedes, 33(89), 73-89.

Calil, E. et Boré, C. (2013). Formas de discurso reportado em narrativas ficcionais escritas por alunos brasileiros e franceses. Debates em Educação, 5, 135-149.

Deschildt, S. (2003). Les paroles de personnages dans les récits des élèves de CM2. Recherches, $39,141-173$.

Doquet, C. L. (2007). Le jeune scripteur et ses doubles. Item, Document téléaccessible à l'adresse: <http://www.item.ens.fr/index.php?id=172883>

Ferreiro, E., Pontecorvo, C., Ribeiro Moreira, N. et Garcia Hidalgo, I. (dir.) (1996). Chapeuzinho Vermelho aprende a escrever: estudos psicolinguísticos comparativos em três línguas. San Paulo: Ática.

Jonasson, K. (2003). Le discours narrativisé dans une vue de Maupassant et sa traduction dans deux versions suédoises. In M. Berré et al. (dir.) La syntaxe raisonnée (p. 297-308). Paris: De Boeck.duculot.

Kellogg, R.T. (2008). Training writing skills: A cognitive developmental perspective. Journal of Writing Research, 1(1), 1-26.

Plane, S., Rondelli, F. et Vénérin, C. (2013). Variations, fidélité, infidélité: l'écriture et la réécriture de discours rapportés par de jeunes scripteurs. In C. Dessoutter et C. Mellet (dir.), Le discours rapporté: approches linguistiques et perspectives didactiques (p. 215-232). Berlin: Peter Lang.

Rosier L. (dir.) (1999). Le discours rapporté. Histoire, théories, pratiques. Paris-Bruxelles: Duculot.

Voloshinov, V. N. (2010). Marxisme et philosophie du langage: les problèmes fondamentaux de la méthode sociologique dans la science du langage. Limoges: Lambert-Lucas.

Zatz, L. et Abreu, G. (2010). O fedor do gambá. In L. Zatz et G. Abreu (dir.) De onde tudo surgiu e como tudo começou (Tudo, tudo mesmo!) (p. 17-21). São Paulo: Moderna. 